\title{
Algebraic or Algorithmic: Searching for Optimal Solutions in Multi-Stage Supply Chain Models
}

\author{
Ab Rahman Ahmad1*, Borkistang Mohamad1, Sami M. Halawani² \\ ${ }^{1}$ Faculty of Computing and Information Technology, King Abdulaziz University, Rabigh, KSA \\ ${ }^{2}$ Faculty of Computing and Information Technology, King Abdulaziz University, Jeddah, KSA \\ Email:^abinahmad@kau.edu.sa,bmohda2000@gmail.com, halawani@kau.edu.sa
}

How to cite this paper: Ahmad, A.R., Mohamad, B. and Halawani, S.M. (2017) Algebraic or Algorithmic: Searching for Optimal Solutions in Multi-Stage Supply Chain Models. Journal of Software Engineering and Applications, 10, 663-676.

https://doi.org/10.4236/jsea.2017.108036

Received: May 16, 2017

Accepted: July 1, 2017

Published: July 4, 2017

Copyright (c) 2017 by authors and Scientific Research Publishing Inc. This work is licensed under the Creative Commons Attribution International License (CC BY 4.0).

http://creativecommons.org/licenses/by/4.0/

\begin{abstract}
In this paper we proposed an $A M H$ Supply Chain model to obtain optimal solutions for Two-, Three- and Four-Stage for deterministic models. Besides deriving its algebraic solutions, a simple searching method is successfully applied in obtaining optimal total costs and its integer multipliers. Our model has shown promising results in comparison to Equal Cycle Time and other existing ones. The tests focused on obtaining optimal total annual costs and other related details of Two-, Three- and Four-Stage for deterministic models. The results are run under Visual Basic Programming platform using Intel ${ }^{\circ}$ Core $^{\mathrm{TM}} 2$ Duo $\mathrm{T} 6500$ Processor.
\end{abstract}

\section{Keywords}

$A M H$ Model, Algebraic Solution, Inventory Coordination, Multi-Stage Supply Chain Models, Simple Search Algorithm

\section{Introduction}

The term Supply Chain Management (SCM) has emerged in the late 1980s and since then many definitions of SCM [1] have been proposed. SCM deals with the managing materials, information and financial flows in a network consisting of suppliers, manufacturers, distributors, wholesalers, retailers and customers (consumers). SCM [2] is defined in as a set of approaches utilized to efficiently integrate suppliers, manufactures, warehouses, and stores, so that merchandise is produced and distributed at the right quantities, to the right locations, and at the right time, in order to minimize system-wide cost while satisfying service level requirements.

There are many different models developed for SCM and planning objectives. The models [3] developed for implementation and application in supply chain management are classified in two mathematical types, i.e., descriptive models 
and optimization models. The descriptive models are created by modeling practitioners to better understanding functional relationship in the supply chain. They include demand forecasting models, cost relationship model, resource utilization relationship models, and supply chain system simulation model.

Research findings in inventory-distribution coordination and information sharing reveal that these can help in reducing inventories, order costs and transportation costs. The firms are advised to device strategies that lead to smaller batches or frequent replenishments. The exchanging substantial quantities of information among the parties involved can increase the efficiency and effectiveness of the supply chain. This can significantly reduced inventory which has a significant impact on supply chain activities.

The focus [4] [5] is on the integrated vendor-buyer inventory and the joint economic lot sizing problem models. The inventories [4] across the entire supply chain can be more efficiently managed through greater cooperation and better coordination. A review in [5] on the joint replenishment problem shows that there is interest in the new kind of problem with dynamic or stochastic demand. Recent research on the problem has also focused on finding faster algorithms to the problem.

The case of a Two-Stage serial supply chain system [6] involves a single vendor who supplies a single buyer with a single product. The vendor's production rate is assumed finite whilst the demand at the buyer is assumed deterministic. The model fully shares the relevant information in order to coordinate the replenishment policies and joint optimize their operational costs. The study developed an integrated inventory replenishment model which assumed linear and fixed backorders costs. A hybrid geometric-algebraic method is used to derive the optimal replenishment policy and the minimum supply chain total cost in a closed form.

Some studies suggested synchronization of cycle times across the chain stages; however, in many cases pure Just-In-Time schedules using a common synchronized common production replenishment cycle are found not to be optimal. The supply chain model [7] achieved better the coordination by applying integer multipliers mechanisms in which the cycle time at each stage is an integer multiple of the cycle time of the adjacent downstream stage.

This research [8] deals with inventory and production co-ordination in a Three-Stage supply chain involving suppliers, manufacturers and retailers. The cycle time used at each stage is an integer multiple of the cycle time of the adjacent downstream stage. The produced lot is sent as soon as they are produced and not to wait until the whole lot is produced. A numerical example shows that the policy leads to better savings compared to the scheme that allows shipments only after the whole lot is produced.

A discrete-event simulation model [9] is developed for a Four-Stage supply chain. The model assumed to share their inventories and demands information. The evaluation on the system performance is the expected total cost which consists of the inventory holding cost, the ordering cost, and the shortage cost. The simulation model is optimized using SimRunner optimization package. 
A more generalized inventory coordination mechanism in an $n$-stage, multi-customer, non-serial supply chain [10] used algebraic method to optimize coordinated supply chain. The established recursive expressions are used for the derivation of the optimal replenishment policy and the development of the solution algorithm. Further, a simple procedure is derived that can help in sharing the coordination cost benefits to induce all stages to adopt the inventory coordination mechanism.

In [11], a proposed formulation for $n$-stage multi customers supply chain inventory model where a company can supply products to several customers. The formulation is for the model with the simplest inventory coordination mechanism, i.e., same cycle time for all companies in the supply chain. Instead of using differential calculus, it is feasible to use perfect square method to obtain an algebraic approach to optimize the supply chain model.

A generalization [7] is performed in [12] and individual derivation of the optimal solution to the Three- and Four-Stage models using the perfect squares method. A simple algebraic approach is considered good for those unfamiliar with differential calculus to understand better the optimal solution procedure. The study also deduced the optimal expressions [7] and [11] via two numerical examples.

The objective for this paper is to introduce our supply chain model called $A M H$, to develop algebraic solutions and performing search algorithms to obtain optimal solutions for multi-stage supply chain models. The development of mathematical formulations can assist the integrated design of strategic supply chain networks. Whenever algebraic formulations are not easily derived, then the alternative is to perform search algorithm. Both results can be utilized to coordinate and integrate the production-inventory decisions efficiently among supply chain partners, for example, suppliers, manufactures, distributors, wholesalers and retailers.

\section{The Notations for Supply Chain Models}

In this paper, up to Four-Stage supply chain partners are considered where a firm can supplies many customers. The production rates for the suppliers, manufactures and distributors are assumed finite and the demand for each firm is assumed to be deterministic. The production and inventory decisions across the supply chain will be coordinated so that the total cost of the system is minimized. The inventory and production cycles in these models are assumed synchronized across the entire supply chain. Figures 1-3 illustrates the examples of Two-, Three- and Four-Stage supply chain model.

The models are developed under the following assumptions:

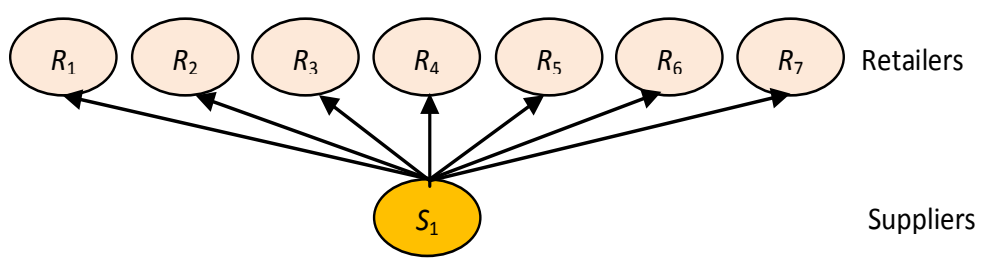

Figure 1. An example of two-stage supply chain model. 
- A single product is produced and distributed up to Four-Stage non-serial multi customers supply chain

- Production rates and demand are deterministic and uniform

- Unsatisfied demands at the end retailers are backordered

- Ordering/setup costs, holding costs are, shortage costs the same for firms at the same stage

- A lot produced at stage is sent in equal shipments to the upstream stage.

The following notations are used in the models developed; where the letters $R$, $D, M$ and $S$ are referred to Retailers, Distributors, Manufactures, and Suppliers.

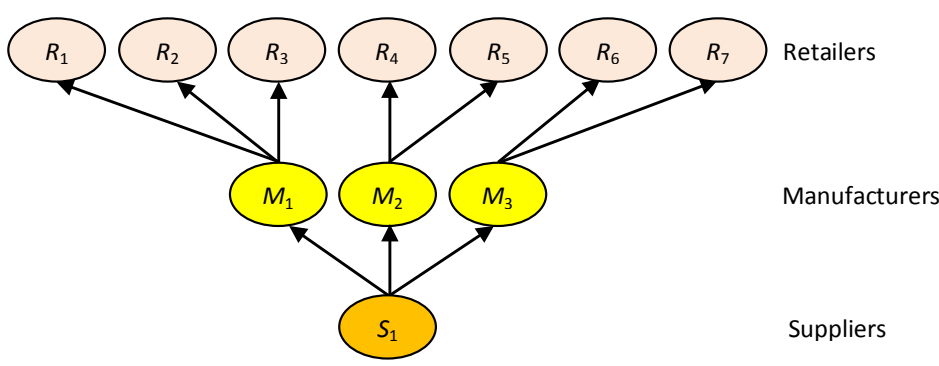

Figure 2. An example of three-stage [7] supply chain model.

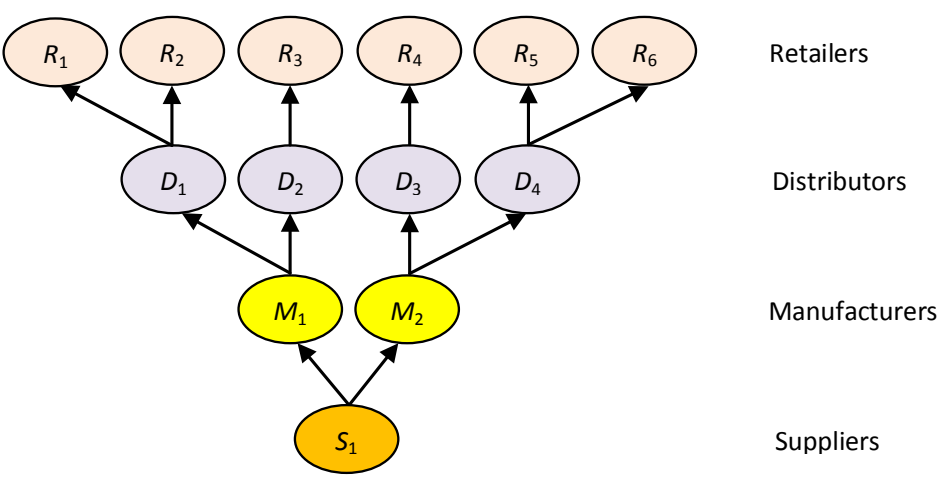

Figure 3. An example of four-stage [10] supply chain model.

\section{$T$}

$T_{R}, T_{D}, T_{M}, T_{S}$

$A_{R}, A_{D}, A_{M}, A_{S}$

$K_{R}, K_{D}, K_{M}, K_{S}$

$2^{K_{R, j}}, 2^{K_{D, j}}, 2^{K_{M, j}}, 2^{K_{S, j}}$

$h_{R}, h_{D}, h_{M}, h_{S}$

$N_{R}, N_{D}, N_{M}, N_{S}$

$D_{R, j}, D_{D, j}, D_{M, j}, D_{S, j}$

$P_{D, j}, P_{M, j}, P_{S, j}$

$\sum_{j} P_{D, j}, \quad \sum_{j} P_{M, j}, \quad \sum_{j} P_{S, j}$

$N_{R} A_{R}, \quad N_{D} A_{D}, \quad N_{M} A_{M}, \quad N_{S} A_{S}$

$\sum_{i, j} D_{R, j}, \sum_{j} D_{D, j}, \sum_{j} D_{M, j}, \sum_{j} D_{s, j}$

$\sum_{j} T C_{R, j}, \sum_{j} T C_{D, j}, \sum_{j} T C_{M, j}, \sum_{j} T C_{s, j}, \sum T C$
Basic cycle time, cycle time at the end retailer

Cycle time at the stages $R, D, M$ and $S$

Setup cost at stages $R, D, M$ and $S$

Integer multiplier at stages $R, D, M$ and $S$

Integer Powers of Two Multipliers of firm $j$ at stages $R, D, M$ and $S$

Inventory holding cost at stages $R, D, M$ and $S$

Number of firms at stages $R, D, M$ and $S$

The Demand Rate of firm $j$ at stages $R, D, M$ and $S$

Production rate of firm $j$ at stages $D, M$ and $S$

The total production rate at stages $D, M$ and $S$

The total setup cost at stages $R, D, M$ and $S$

The total demand rate at stages $R, D, M$ and $S$

The total cost for $R, D, M$ and $S$ and the entire supply chain 
The total annual cost for a downstream firm consist of

$$
\sum T C=\text { Cost of keeping products }+ \text { reorder cost }
$$

For example, the total annual cost for retailers is given by

$$
\sum_{j} T C_{R, j}=\sum_{j}\left(\frac{T D_{R, j} h_{R}}{2}+\frac{A_{R}}{T}\right) .
$$

For a firm involves in making products, we can express it in three parts

$\sum T C=$ Cost of making product + cost of keeping finished product + setup cost

For example, the total annual cost for manufacturers is

$$
\sum_{j} T C_{M, j}=\sum_{j}\left(\frac{T D_{M, j}^{2}}{2 P_{M, j}}\left(h_{S}+h_{M}\right)+\frac{A_{M}}{T}\right) .
$$

The variation on the cost of keeping finished products can determine the lowest annual cost.

We present a deterministic Four-Stage supply chain model formulated for two inventory coordination mechanisms-Equal Cycle Time (ECT) and Integer Multipliers (IM). The mathematical formulation for Two- and Three-Stage can be derived accordingly by reducing the model into required stage. Under the configuration, a firm can supply any customer in the adjacent upstream stage.

\section{Four-Stage Supply Chain Models}

Four-Stage supply chain model example based on [10] is depicted in Figure 3. The chain consists of a supplier, two manufacturers, four distributors and six retailers.

First, we discuss the coordination mechanisms, i.e., the equal cycle time and the integer multipliers. Let $T$ is the basic cycle time. In Equal Cycle Time (ECT) mechanism, all firms in the chain have similar cycle time $T$. In contrast, for the Integer Multipliers (IM) mechanism models firms, each stage use the same cycle time but the cycle time at each stage is multiplied by an integer of the cycle time at the adjacent downstream stage. This implies that $T_{D}=K_{D} T, T_{M}=K_{M} K_{D} T$ and $T_{S}=K_{S} K_{M} K_{D} T$ where $K_{D}, K_{M}, K_{S}$ and $T_{D}, T_{M}, T_{S}$ are the $I M$ and cycle times for Distributors, Manufacturers and Suppliers respectively.

\subsection{Equal Cycle Time (ECT)}

Under $E C T$, the total annual cost for four-stage model is summarized as Equation (1)

$$
\begin{aligned}
\sum T C= & \sum_{j=1}^{N_{R}}\left(\frac{T D_{R, j}}{2} h_{R}+\frac{A_{R}}{T}\right)+\sum_{j=1}^{N_{D}}\left(\frac{T D_{D, j}^{2}}{2 P_{D, j}}\left(h_{M}+h_{D}\right)+\frac{A_{D}}{T}\right) \\
& +\sum_{j=1}^{N_{M}}\left(\frac{T D_{M, j}^{2}}{2 P_{M, j}}\left(h_{S}+h_{M}\right)+\frac{A_{M}}{T}\right)+\sum_{j=1}^{N_{S}}\left(\frac{T D_{S, j}^{2}}{2 P_{S, j}}\left(h_{0}+h_{S}\right)+\frac{A_{S}}{T}\right) \\
= & \frac{T}{2}\left[\sum_{j=1}^{N_{R}} D_{R, j} h_{R}+\sum_{j=1}^{N_{D}} \frac{T D_{D, j}^{2}}{P_{D, j}}\left(h_{M}+h_{D}\right)+\sum_{j=1}^{N_{M}} \frac{T D_{M, j}^{2}}{P_{M, j}}\left(h_{S}+h_{M}\right)\right. \\
& \left.+\sum_{j=1}^{N_{S}} \frac{T D_{S, j}^{2}}{P_{S, j}}\left(h_{0}+h_{S}\right)\right]+\frac{1}{T}\left(N_{R} A_{R}+N_{D} A_{D}+N_{M} A_{M}+N_{S} A_{S}\right)
\end{aligned}
$$


Let

$W=\frac{T}{2}\left[\sum_{j=1}^{N_{R}} D_{R, j} h_{R}+\sum_{j=1}^{N_{D}} \frac{T D_{D, j}^{2}}{P_{D, j}}\left(h_{M}+h_{D}\right)+\sum_{j=1}^{N_{M}} \frac{T D_{M, j}^{2}}{P_{M, j}}\left(h_{S}+h_{M}\right)+\sum_{j=1}^{N_{S}} \frac{T D_{S, j}^{2}}{P_{S, j}}\left(h_{0}+h_{S}\right)\right]$

and $Y=\frac{1}{T}\left(N_{R} A_{R}+N_{D} A_{D}+N_{M} A_{M}+N_{S} A_{S}\right)$.

The algebraic solution for the $T$ and $T C$ can be obtained using simple procedure as follows:

With $T C=W T+\frac{Y}{T}$, then $\frac{\mathrm{d} T C}{\mathrm{~d} t}=W-\frac{Y}{T^{2}}$.

Equating $\frac{\mathrm{d} T C}{\mathrm{~d} t}=0$, we then have $W-\frac{Y}{T^{2}}=0$, thus giving the solution as Equation (2)

$$
T_{\text {opt }}=\sqrt{\frac{Y}{W}} \text { and } T C_{\text {opt }}=2 \sqrt{W Y}
$$

\subsection{Khouja's Model}

During non-production times [7], the distributors' inventory drop every $T$ years by $T D_{D, j}$ which reduce the annual holding cost to $0.5\left(K_{D}-1\right) T D_{D_{, j}} h_{D}$. The same applies to manufacturers and suppliers where the costs are reduced to $0.5 K_{D}\left(K_{M}-1\right) T D_{M, j} h_{M}$ and $0.5 K_{D} K_{M}\left(K_{S}-1\right) T D_{S, j} h_{S}$ respectively. Thus, we obtain Equation (3) as follows

$$
\begin{aligned}
\sum T C= & \sum_{j=1}^{N_{R}}\left(\frac{T D_{R, j}}{2} h_{R}+\frac{A_{R}}{T}\right)+\sum_{j=1}^{N_{D}}\left(\frac{K_{D} T D_{D, j}^{2}}{2 P_{D, j}}\left(h_{M}+h_{D}\right)+\frac{\left(K_{D}-1\right) T D_{D, j}}{2} h_{D}+\frac{A_{D}}{K_{D} T}\right) \\
& +\sum_{j=1}^{N_{M}}\left(\frac{K_{D} K_{M} T D_{M, j}^{2}}{2 P_{M, j}}\left(h_{S}+h_{M}\right)+\frac{K_{D}\left(K_{M}-1\right) T D_{M, j}}{2} h_{M}+\frac{A_{M}}{K_{D} K_{M} T}\right) \\
& +\sum_{j=1}^{N_{S}}\left(\frac{K_{D} K_{M} K_{S} T D_{S, j}^{2}}{2 P_{S, j}}\left(h_{0}+h_{S}\right)+\frac{K_{D} K_{M}\left(K_{S}-1\right) T D_{S, j}}{2} h_{S}+\frac{A_{S}}{K_{D} K_{M} K_{S} T}\right)
\end{aligned}
$$

Rearranging Equation (3) as Equation (4), we have

$$
\begin{aligned}
\sum T C= & \frac{T}{2}\left[K _ { D } \left[K _ { M } \left(K_{S} \sum_{j=1}^{N_{S}}\left(\frac{D_{S, j}^{2}}{P_{S, j}}\left(h_{0}+h_{S}\right)+D_{S, j} h_{S}\right)\right.\right.\right. \\
& \left.+\sum_{j=1}^{N_{M}}\left(\frac{D_{M, j}^{2}}{P_{M, j}}\left(h_{S}+h_{M}\right)+D_{M, j} h_{M}\right)-\sum_{j=1}^{N_{S}} D_{S, j} h_{S}\right) \\
& \left.+\sum_{j=1}^{N_{D}}\left(\frac{D_{D, j}^{2}}{P_{D, j}}\left(h_{M}+h_{D}\right)+D_{D, j} h_{D}\right)-\sum_{j=1}^{N_{M}} D_{M, j} h_{M}\right]+\sum_{j=1}^{N_{R}} D_{R, j} h_{R} \\
& \left.-\sum_{j=1}^{N_{D}} D_{D, j} h_{D}\right]+\frac{1}{T}\left(N_{R} A_{R}+\frac{N_{D} A_{D}}{K_{D}}+\frac{N_{M} A_{M}}{K_{D} K_{M}}+\frac{N_{S} A_{S}}{K_{D} K_{M} K_{S}}\right)
\end{aligned}
$$

and let

$$
\alpha_{D}=\sum_{j=1}^{N_{D}}\left(\frac{D_{D, j}^{2}}{P_{D, j}}\left(h_{M}+h_{D}\right)+D_{D, j} h_{D}\right), \quad \alpha_{M}=\sum_{j=1}^{N_{M}}\left(\frac{D_{M, j}^{2}}{P_{M, j}}\left(h_{S}+h_{M}\right)+D_{M, j} h_{M}\right)
$$




$$
\begin{gathered}
\alpha_{S}=\sum_{j=1}^{N_{S}}\left(\frac{D_{S, j}^{2}}{P_{S, j}}\left(h_{0}+h_{S}\right)+D_{S, j} h_{S}\right), \beta_{R D}=\sum_{j=1}^{N_{R}} D_{R, j} h_{R}-\sum_{j=1}^{N_{D}} D_{D, j} h_{D} \\
\beta_{M}=\sum_{j=1}^{N_{M}} D_{M, j} h_{M}, \beta_{S}=\sum_{j=1}^{N_{S}} D_{S, j} h_{S}, \\
\omega=\left(N_{R} A_{R}+\frac{N_{D} A_{D}}{K_{D}}+\frac{N_{M} A_{M}}{K_{D} K_{M}}+\frac{N_{S} A_{S}}{K_{D} K_{M} K_{S}}\right) \\
\omega=N_{R} A_{R}+\frac{\varphi_{D}}{K_{D}} \text { and } \varphi_{D}=N_{D} A_{D}+\frac{N_{M} A_{M}}{K_{M}}+\frac{N_{S} A_{S}}{K_{M} K_{S}}, \varphi_{M}=N_{M} A_{M}+\frac{N_{S} A_{S}}{K_{S}} \\
\sum T C=T\left[\frac{K_{D}}{2}\left[K_{M}\left(K_{S} \alpha_{S}+\alpha_{M}-\beta_{S}\right)+\alpha_{D}-\beta_{M}\right]+\beta_{R D}\right]+\frac{\omega}{T} . \text { Let, } \\
W=\frac{K_{D}}{2}\left(K_{M}\left(K_{S} \alpha_{S}+\alpha_{M}-\beta_{S}\right)+\alpha_{D}-\beta_{M}\right)+\beta_{R D}, Y=\omega .
\end{gathered}
$$

Differentiating $\frac{\mathrm{d} T C}{\mathrm{~d} T}$ then solving for $\frac{\mathrm{d} T C}{\mathrm{~d} T}=0$, we obtain Equation (6)

$$
\begin{aligned}
& T=\sqrt{\frac{2 \omega}{K_{D}\left[K_{M}\left(K_{S} \alpha_{S}+\alpha_{M}-\beta_{S}\right)+\alpha_{D}-\beta_{M}\right]+\beta_{R D}}} \\
& T C=\sqrt{2\left[K_{D}\left[K_{M}\left(K_{S} \alpha_{S}+\alpha_{M}-\beta_{S}\right)+\alpha_{D}-\beta_{M}\right]+\beta_{R D}\right] \omega} .
\end{aligned}
$$

Next, differentiating and solving for $\frac{\mathrm{d} T C}{\mathrm{~d} K_{D}}=0, \frac{\mathrm{d} T C}{\mathrm{~d} K_{M}}=0$ and $\frac{\mathrm{d} T C}{\mathrm{~d} K_{S}}=0$ we then have Equation (7)

$$
\begin{aligned}
& K_{S}^{o p t}=\sqrt{\frac{\left(\alpha_{M}-\beta_{S}\right) N_{S} A_{S}}{\alpha_{S} N_{M} A_{M}}}, \quad K_{M}^{o p t}=\sqrt{\frac{\left(\alpha_{D}-\beta_{M}\right) \varphi_{M}}{\left(K_{S} \alpha_{S}+\alpha_{M}-\beta_{S}\right) N_{D} A_{D}}} \\
& K_{D}^{o p t}=\sqrt{\frac{\beta_{R D} \varphi_{D}}{\left[K_{M}\left(K_{S} \alpha_{S}+\alpha_{M}-\beta_{S}\right)+\alpha_{D}-\beta_{M}\right] N_{R} A_{R}}} .
\end{aligned}
$$

Substituting (7) in (6), gives us $T_{\text {opt }}$ and $T C_{\text {opt }}$.

\subsection{Our Proposed Model-AMH}

Our proposed model $A M H$-Ahmad, Mohamad and Halawani is an improvement based on Three-Stage model [8]. Instead of using both algebraic and searching algorithm, we will show our model requires only a mathematical formulation in obtaining the results.

In this model, goods produced at any stage are delivered to the upstream stage in equal shipments as they are produced and depend on the cycle time of the stage. In 4-Stage model, the inventory level of distributors at the beginning is at its minimum level which is equal to $D_{D, j}^{2} / P_{D, j}$. Then it starts to increase at the rate of $P_{D, j}-D_{D, j}$ until it reaches its peak at $\left(P_{D, j}-D_{D, j}\right) K_{D} T D_{D, j} / P_{D, j}$. Then it is consumed at the rate $D_{D, j}$ until the end of the cycle.

For manufacturers and suppliers, their respective inventory levels reach their peaks at $\left(P_{M, j}-D_{M, j}\right) K_{D} K_{M} T D_{M, j} / P_{M, j}$ and $\left(P_{S, j}-D_{S, j}\right) K_{D} K_{M} K_{S} T D_{S, j} / P_{S, j}$. These then consumed at the respective rate of $K_{D} D_{M, j}$ and $K_{D} K_{M} D_{S, j}$ until 
the end of the cycle. The total annual cost for Four-Stage $A M H$ model can be summarized as Equation (8)

$$
\begin{aligned}
\sum T C= & \sum_{j=1}^{N_{R}}\left(\frac{T D_{R, j}}{2} h_{R}+\frac{A_{R}}{T}\right)+\sum_{j=1}^{N_{D}}\left(\frac{K_{D} T D_{D, j}^{2}}{2 P_{D, j}}\left(h_{M}+h_{D}\right)+\left[\frac{\left(P_{D, j}-D_{D, j}\right) K_{D} T D_{D, j}}{2 P_{D, j}}-\frac{T D_{D, j}}{2}\right] h_{D}+\frac{A_{D}}{K_{D} T}\right) \\
& +\sum_{j=1}^{N_{M}}\left(\frac{K_{D} K_{M} T D_{M, j}^{2}}{2 P_{M, j}}\left(h_{S}+h_{M}\right)+K_{D}\left[\frac{\left(P_{M, j}-D_{M, j}\right) K_{M} T D_{M, j}}{2 P_{M, j}}-\frac{T D_{M, j}}{2}\right] h_{M}+\frac{A_{M}}{K_{D} K_{M} T}\right) \\
& +\sum_{j=1}^{N_{S}}\left(\frac{K_{D} K_{M} K_{S} T D_{S, j}^{2}}{2 P_{S, j}}\left(h_{0}+h_{S}\right)+K_{D} K_{M}\left[\frac{\left(P_{S, j}-D_{S, j}\right) K_{S} T D_{S, j}}{2 P_{S, j}}-\frac{T D_{S, j}}{2}\right] h_{S}+\frac{A_{S}}{K_{D} K_{M} K_{S} T}\right)
\end{aligned}
$$

Rearranging Equation (8) as Equation (9), we obtain

$$
\begin{aligned}
\sum T C= & \frac{T}{2}\left[K _ { D } \left(K _ { M } \left(K_{S} \sum_{j=1}^{N_{S}}\left(\frac{D_{S, j}^{2}}{P_{S, j}}\left(h_{0}+h_{S}\right)+\frac{\left(P_{S, j}-D_{S, j}\right) h_{S} D_{S, j}}{P_{S, j}}\right)\right.\right.\right. \\
& \left.+\sum_{j=1}^{N_{M}}\left(\frac{D_{M, j}^{2}}{P_{M, j}}\left(h_{S}+h_{M}\right)+\frac{\left(P_{M, j}-D_{M, j}\right) h_{M} D_{M, j}}{P_{M, j}}\right)-\sum_{j=1}^{N_{S}} h_{S} D_{S, j}\right) \\
& \left.+\sum_{j=1}^{N_{D}}\left(\frac{D_{D, j}^{2}}{P_{D, j}}\left(h_{M}+h_{D}\right)+\frac{\left(P_{D, j}-D_{D, j}\right) h_{D} D_{D, j}}{P_{D, j}}\right)-\sum_{j=1}^{N_{M}} h_{M} D_{M, j}\right) \\
& \left.+\sum_{j=1}^{N_{R}} D_{R, j} h_{R}-\sum_{j=1}^{N_{D}} h_{D} D_{D, j}\right]+\frac{1}{T}\left(N_{R} A_{R}+\frac{N_{D} A_{D}}{K_{D}}+\frac{N_{M} A_{M}}{K_{D} K_{M}}+\frac{N_{S} A_{S}}{K_{D} K_{M} K_{S}}\right)
\end{aligned}
$$

and let

$$
\begin{gathered}
\alpha_{D}=\sum_{j=1}^{N_{D}}\left(\frac{D_{D, j}^{2}}{P_{D, j}}\left(h_{M}+h_{D}\right)+\frac{\left(P_{D, j}-D_{D, j}\right) h_{D} D_{D, j}}{P_{D, j}}\right), \quad \beta_{M}=\sum_{j=1}^{N_{M}} h_{M} D_{M, j} \\
\alpha_{M}=\sum_{j=1}^{N_{M}}\left(\frac{D_{M, j}^{2}}{P_{M, j}}\left(h_{S}+h_{M}\right)+\frac{\left(P_{M, j}-D_{M, j}\right) h_{M} D_{M, j}}{P_{M, j}}\right), \quad \beta_{R D}=\sum_{j=1}^{N_{R}} D_{R, j} h_{R}-\sum_{j=1}^{N_{D}} h_{D} D_{D, j} \\
\alpha_{S}=\sum_{j=1}^{N_{S}}\left(\frac{D_{S, j}^{2}}{P_{S, j}}\left(h_{0}+h_{S}\right)+\frac{\left(P_{S, j}-D_{S, j}\right) h_{S} D_{S, j}}{P_{S, j}}\right), \quad \beta_{S}=\sum_{j=1}^{N_{S}} h_{S} D_{S, j} \\
\omega=N_{R} A_{R}+\frac{N_{D} A_{D}}{K_{D}}+\frac{N_{M} A_{M}}{K_{D} K_{M}}+\frac{N_{S} A_{S}}{K_{D} K_{M} K_{S}}, \omega=N_{R} A_{R}+\frac{\varphi_{D}}{K_{D}} \\
\varphi_{D}=N_{D} A_{D}+\frac{N_{M} A_{M}}{K_{M}}+\frac{N_{S} A_{S}}{K_{M} K_{S}}, \text { and } \varphi_{M}=N_{M} A_{M}+\frac{N_{S} A_{S}}{K_{S}} \\
\sum T C=\frac{T}{2}\left[K_{D}\left(K_{M}\left(K_{S} \alpha_{S}+\alpha_{M}-\beta_{S}\right)+\alpha_{D}-\beta_{M}\right)+\beta_{R D}\right]+\frac{\omega}{T} . \text { Let, } \\
W=\frac{K_{D}}{2}\left(K_{M}\left(K_{S} \alpha_{S}+\alpha_{M}-\beta_{S}\right)+\alpha_{D}-\beta_{M}\right)+\beta_{R D}, \quad Y=\omega,
\end{gathered}
$$

which is similar to Equation (5). Using (7) and (6), we can obtain $K_{S}^{\text {opt }}, K_{M}^{\text {opt }}$, $K_{D}^{o p t}, T_{\text {opt }}$ and $T C_{\text {opt }}$. 


\section{Searching Algorithm}

Algebraic solutions to some mathematical problems can be derived to obtain the intended results instantly. However, some difficult expressions might require the solutions to be obtained iteratively or by approximation. Three-Stage Model [8] for example, is first solved by mathematical formulation then requires a method of searching for its final solution. For this purpose, we devise a simple searching method that can provide solutions as accurate as algebraic counterparts.

Equations (1), (4) and (9) can be written in the form of Equation (10)

$$
T C=W T+\frac{Y}{T}
$$

where $W$ and $Y$ are constants and positive. This gives $T C>0$ and depends on $T$.

To illustrates this, Figure 4 shows an example for $T C=W T+Y / T$ where $W=$ 1 and $Y=20$. The blue line (straight) $Y_{1}=T$ and the red hyperbola (curve) $Y_{2}=20 / T$ meets to yield an optimal annual cost, $T C_{\text {opt }}$ on the green line function TC.

A simple search to determine $T_{o p t}$ and $T C_{o p t}$ based on Figure 5 is presented as follows. First, an initial value for $T C_{o p t}$ is required prior to perform the search. Let $T, T_{S}, T_{E}$ and $T_{A}$ are the respective cycle time, its start, end and accuracy that create the main loop. Since the bounds of cycle time $T$ is $(0,1)$, then we can select $T_{S}=0.01$ and $T_{E}=0.99$. For $T_{A}$, the good choice is within 0.001 to 0.00001 ( 3 to 5 decimal places accuracy).

The main loop is sufficient for Equation (1); however Equations (4) and (9) involve integer multipliers which require more calculations. In addition to the loop created by cycle time $T$, the number of multipliers used in $I M$ mechanisms generates more loops. The bounds set for these mechanisms determine the number of repetition required. For Four-Stage $I M$, the number of loops generated are $L_{D} \times L_{M} \times L_{S}$ where $L_{D}, L_{M}$ and $K_{S}$ are the respective limits for

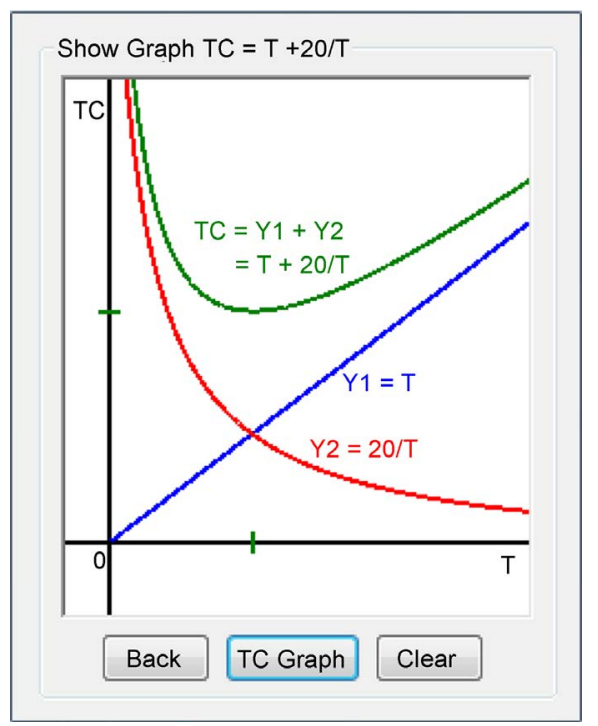

Figure 4. The sketch of an optimal solution for $T_{\text {opt }}$ and $T C_{o p t}$. 


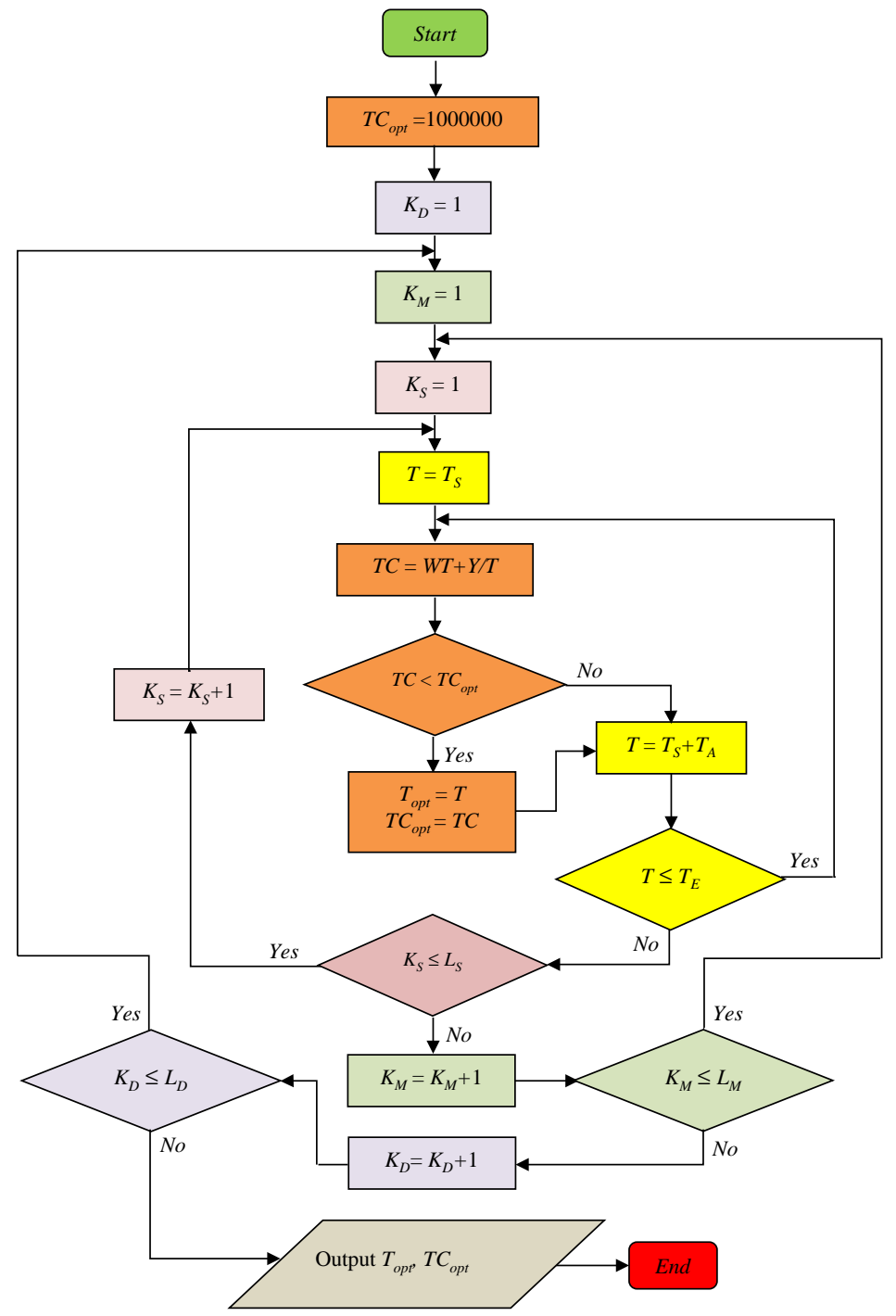

Figure 5. Searching for an optimal solution for $T_{\text {opt }}$ and $T C_{o p t}$.

$K_{D}, K_{M}$ and $K_{S}$. When these limits are reached the loops terminate, giving us $T_{\text {opt }}, T C_{\text {opt }}$ and the optimal values of multipliers.

We present the flowchart for Searching for an Optimal Solution for $T_{o p t}$ and $T C_{\text {opt }}$ for Four-Stage $I M$ model as depicted in Figure 5.

For Three-Stage $I M$ model skips $K_{D}$; for Two-Stage $I M$ model skips $K_{D}$ and $K_{M}$; and for Multi-Stage ECT model skips $K_{D}, K_{M}$ and $K_{S}$.

\section{Results and Discussion}

Figures 6-8 are the results for the respective Two-, Three- and Four-Stage models run on Visual Basic Programming platform. The sample data [7] is used for Two- and Three-Stage (Figure 6 and Figure 7) whilst for Four-Stage (Figure 8) we have considered the sample [9]. Figure 9 shows the nature of total costs where the optimal value for each stage and mechanism exists.

For Three-Stage model as shown in Figure 7, our model $(A M H)$ yields similar results as [8] at 45,987 but the advantage is we are able to solve it algebraically or 


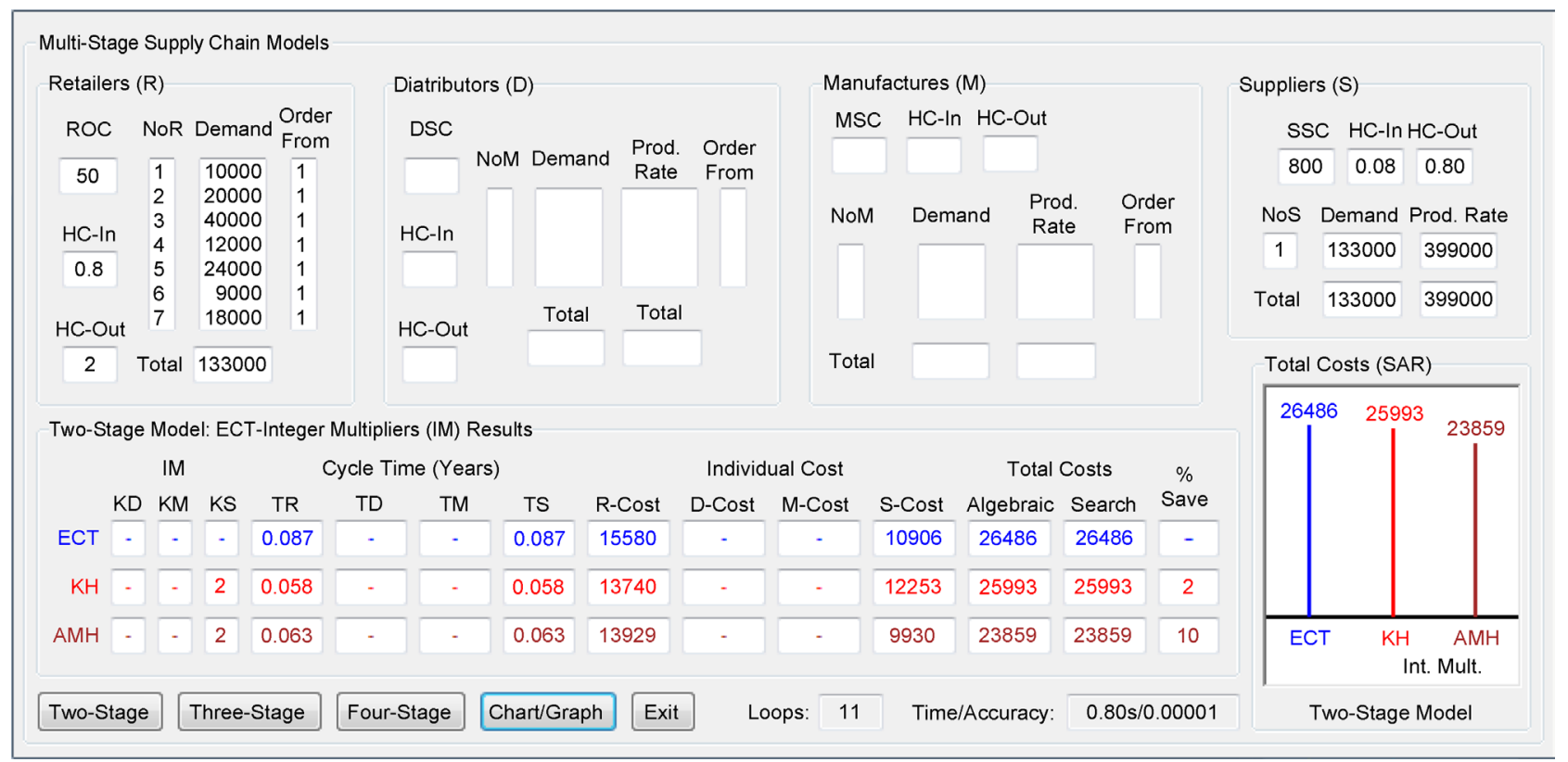

Figure 6. The detail results for two-stage supply chain models.

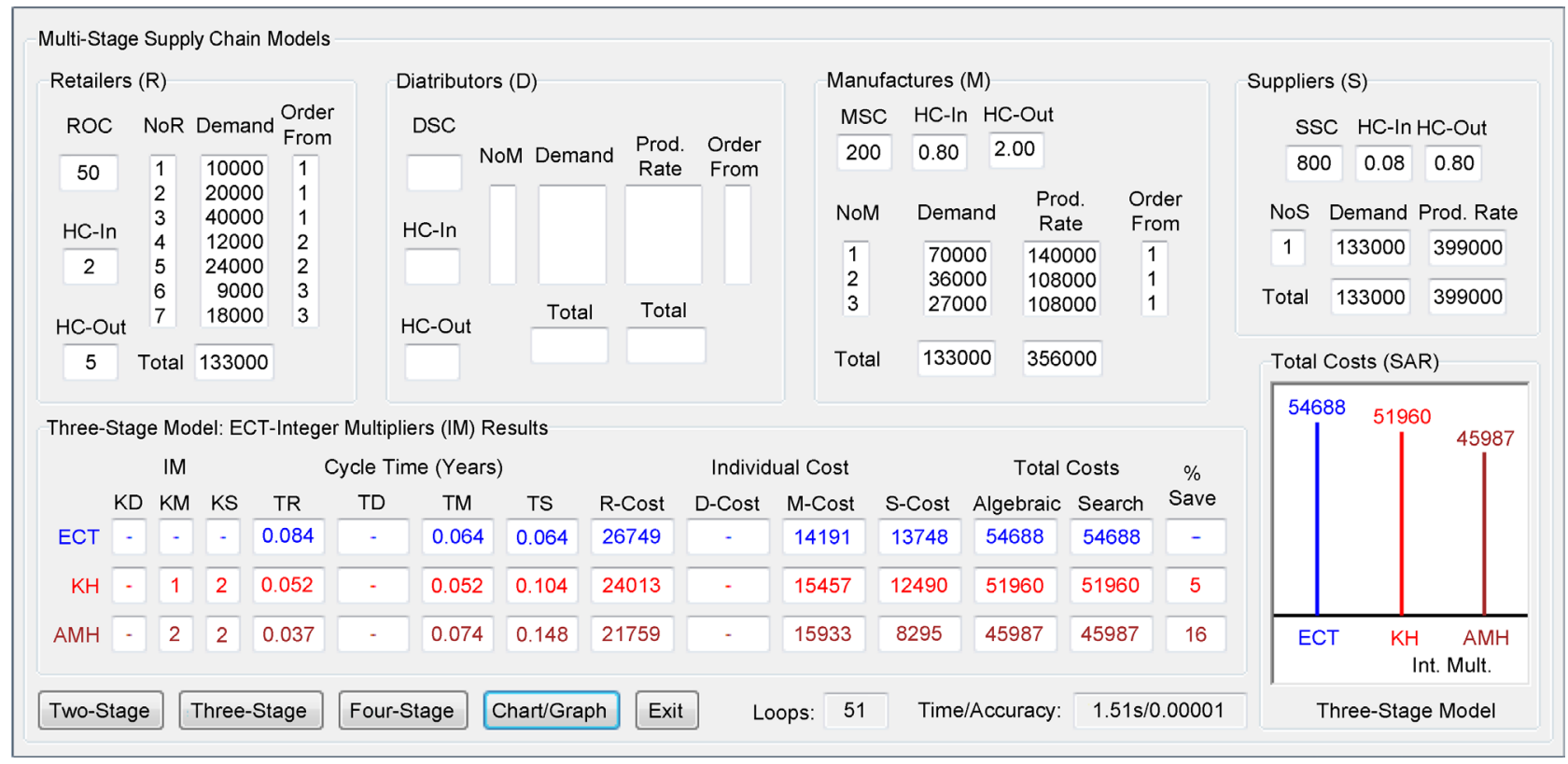

Figure 7. The detail results for three-stage supply chain models.

via searching. A better improvement over $E C T$ and [7] is clear, both at 54,688 and 51,960 respectively. Another saving is also shown in Two-Stage model (Figure 6), where the total cost for $A M H$ is 23,859 in comparison to 26,486 and 25,933. More convincing result is shown further in Four-Stage model, Figure 8. $A M H$ shows very much further reduction with the total cost of 51,400 . The saving over $E C T$ for three different stages is $10 \%, 16 \% 35 \%$ compared to $K H$ of $2 \%$, $5 \%$ and $25 \%$.

Figure 9 displays a clear behavior of total costs $T C=W T+Y / T, W, Y>0$ for each model and mechanism. 


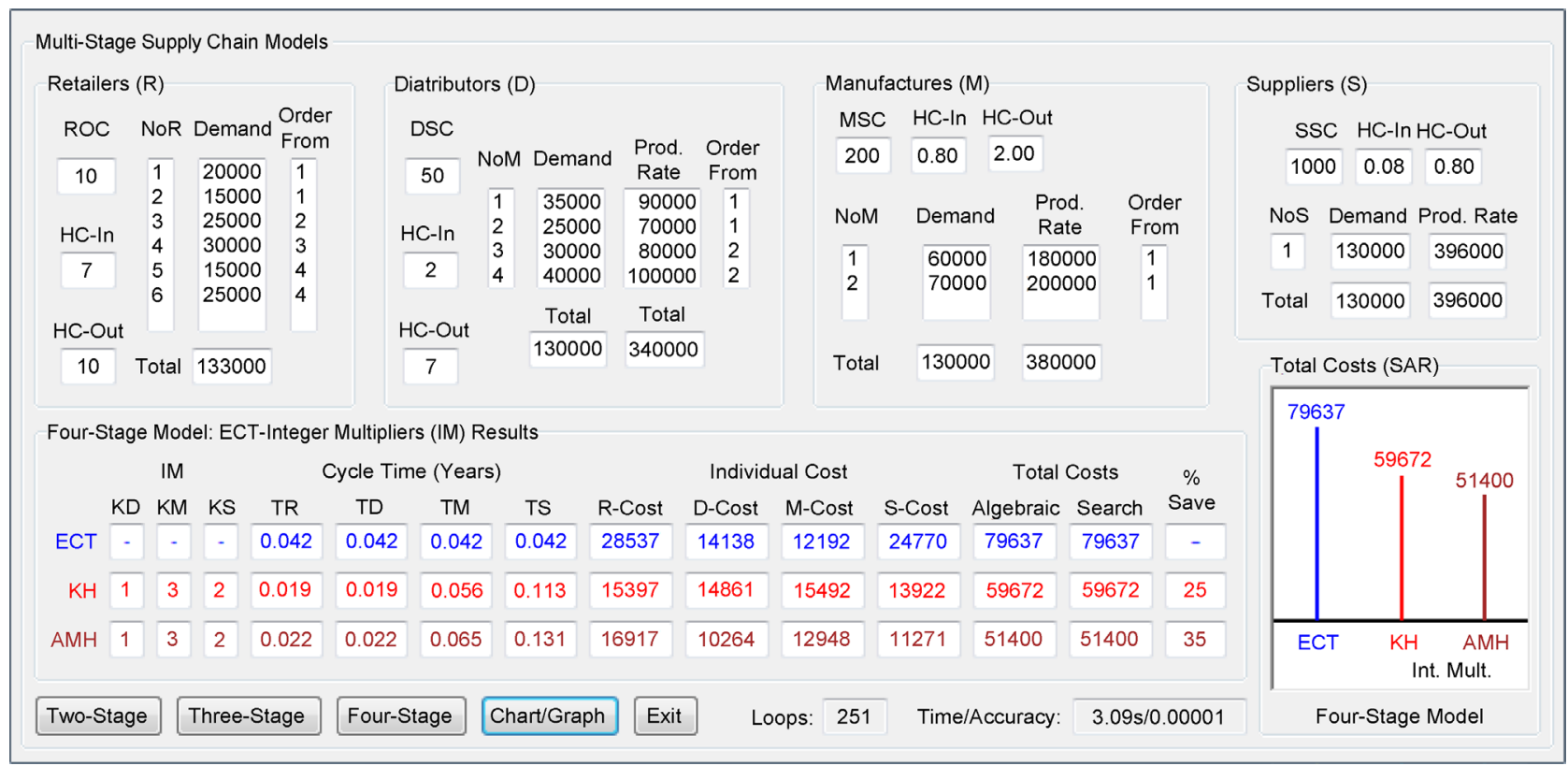

Figure 8. The detail results for four-stage supply chain models.
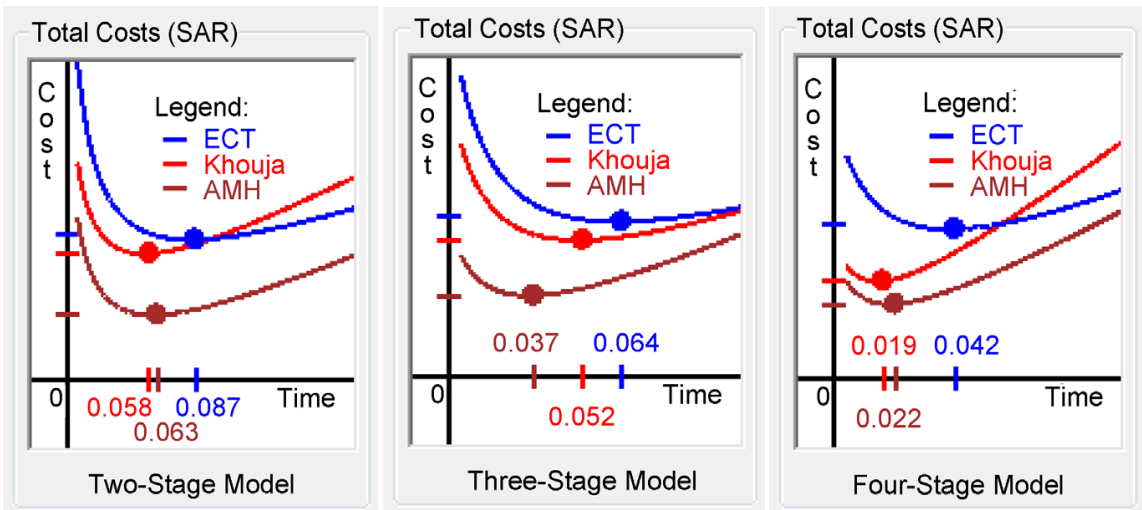

Figure 9. The graph for two, three and four-stage supply chain models.

These significant gains in all cases are due to proper handling of finished products within non-production activities. These products are shipped as soon as they are produced, thus saving the holding cost drastically. This can be seen from the reduction in individual cost for distributors, manufacturers and suppliers in Figure 8 for each mechanism.

Algebraically, it has been shown in each case that there exists only one optimal value. The dots represent the optimal value for the mechanisms in each model. This is similar to the chart depicted in Figures 6-8 which shows the evidence that $A M H$ is a far better scheme over ECT and [7]. The searching technique used also yields the same results as the algebraic solutions as shown in Figures 6-8.

For searching algorithm, the time taken for Two-, Three- and Four-Stage are measured in second. Their respective times are 0.81, 1.51 and 3.09 seconds and running under Inter ${ }^{\oplus} \mathrm{Core}^{\mathrm{TM}} 2$ duo $\mathrm{T} 6500$ Processor. The latest processor with faster speed would reduce further the time required to produce the results. It is clear that the results are obtainable via mathematical formulation or perform 
searching algorithm.

\section{Conclusions}

To date, supply chains are spanning thousands of miles across the globe involving numerous suppliers, manufacturers, distributors, wholesalers, dealers, retailers and finally to customers (consumers). All are important in each of their scopes and interdependent on one another. It is consumers who create the demand based on manufacturers' goods produced. If consumers reduce the consumption of a particular product, its supply also has to be reduced to balance the demand.

In this paper we have developed $A M H$ model to obtain optimal solutions for Two-, Three- and Four-Stage Supply Chain models. Our $A M H$ model has shown promising results in comparison to $E C T$ and [7]. Besides deriving algebraic solutions, a simple searching method is successfully applied in obtaining optimal total costs and its integer multipliers.

The model is just tested on deterministic demand; however, this can be extended to deal with other issues, variations and constraints such as stochastic demand or other costs incurred. Developing a computerized system that can handle transactions in small or medium business is feasible. The system should be able to perform some required tasks in simple Two-Stage to bigger N-Stage models that involve more firms and with the variation in supply chain management.

\section{Acknowledgements}

This project was funded by the Deanship of Scientific Research (DSR), King Abdulaziz University, Jeddah under grant no 499/830/1431. The authors, therefore, acknowledge with thanks to DSR technical and financial support.

\section{References}

[1] Nahmias, S. (2005) Production and Operations Analysis. 5th Edition, McGrawHill/Irwin, Singapore.

[2] Simchi-Levi, D., Kaminsky, P. and Simchi-Levi, E. (2008) Designing \& Managing the Supply Chain: Concepts, Strategies, and Case Studies. 3rd Edition, McGraw Hill/Irwin, Boston.

[3] Shapiro, J.F. (2009) Modeling the Supply Chain. 2nd Edition, Cengage Learning, Duxbury, MA.

[4] Ben-Daya, M., Darwish, M. and Ertogral, K. (2008) The Joint Economic Lot Sizing Problem: Review and Extensions. European Journal of Operational Research, 185, 726-742. https://doi.org/10.1016/j.ejor.2006.12.026

[5] Khouja, M. and Goyal, S. (2008) A Review of the Joint Replenishment Problem Literature: 1989-2005. European Journal of Operational Research, 186, 1-16. https://doi.org/10.1016/j.ejor.2007.03.007

[6] Seliaman, M.E. (2013) Optimizing the Two-Stage Supply Chain Inventory Model with Full Information Sharing and Two Backorders Costs Using Hybrid Geometric-Algebraic Method. Journal of Optimization, 5 p. 
https://doi.org/10.1155/2013/519180

[7] Khouja, M. (2003) Optimizing Inventory Decisions in a Multi-Stage Multi-Customer Supply Chain. Transportation Research Part E Logistics and Transportation Review, 39, 193-208. https://doi.org/10.1016/S1366-5545(02)00036-4

[8] Ben-Daya, M. and Al-Nassar, A. (2008) An Integrated Inventory Production System in a Three-Layer Supply Chain. Journal of Production Planning and Control, 19, 97-104. https://doi.org/10.1080/09537280801895930

[9] Saif, A.-W.A., Seliaman, M.E. and Ahmad A.R. (2006) Simulation Optimization of a Four-Stage Supply Chain System. IEEE $2^{\text {nd }}$ Information and Communication Technologies, 2006. ICTTA 06, Damascus, 24-28 April 2006, 2798-2802. https://doi.org/10.1109/ictta.2006.1684855

[10] Seliaman, M.E. and Ahmad, A.R. (2009) A Generalized Algebraic Model for Optimizing Inventory Decisions in A Multi-Stage Complex Supply Chain. Transportation Research Part E, 45, 409-418. https://doi.org/10.1016/j.tre.2008.09.012

[11] Cárdenas-Barrón, L.E. (2007) Optimizing Inventory Decisions in a Multi-Stage Multi-Customer Supply Chain: A Note. Transportation Research Part E, 43, 647654. https://doi.org/10.1016/j.tre.2005.09.011

[12] Leung, K.N.F. (2009) A Technical Note on Optimizing Inventory Decisions in a Multi-Stage Multi-Customer Supply Chain. Transportation Research Part E, 45, 572-582. https://doi.org/10.1016/j.tre.2009.01.007

Submit or recommend next manuscript to SCIRP and we will provide best service for you:

Accepting pre-submission inquiries through Email, Facebook, LinkedIn, Twitter, etc. A wide selection of journals (inclusive of 9 subjects, more than 200 journals) Providing 24-hour high-quality service User-friendly online submission system Fair and swift peer-review system Efficient typesetting and proofreading procedure Display of the result of downloads and visits, as well as the number of cited articles Maximum dissemination of your research work

Submit your manuscript at: http://papersubmission.scirp.org/

Or contact jsea@scirp.org 